\title{
Prevalência de sobrepeso e obesidade em crianças de seis a dez anos de escolas municipais de área urbana
}

\author{
Prevalence of overweight and obesity in six to ten year-old students from urban county schools
}

\author{
Anne Dal M. Mello ${ }^{1}$, Sonia Silva Marcon ${ }^{2}$, Ana Paula C. R. Hulsmeyer ${ }^{3}$, Glauco Barnez P. Cattai ${ }^{4}$, Carla Simara L. S. Ayres 5 , \\ Rosangela Getirana Santana ${ }^{6}$
}

\section{RESUMO}

Objetivo: Identificar a prevalência de sobrepeso e obesidade e fatores associados em alunos de seis a dez anos das escolas públicas municipais da área urbana de Marialva, no Paraná.

Métodos: Estudo transversal que utilizou uma amostra aleatória estratificada de 356 alunos. Foram avaliadas as seguintes variáveis: índice de massa corpórea, sexo, faixa etária, número de irmãos, atividade física e atividades sedentárias. Utilizou-se estatística descritiva para apresentação da distribuição da amostra nas estratificações. O teste do qui-quadrado foi aplicado para verificar a diferença entre a distribuição da amostra para cada uma das categorias das variáveis. A associação entre as variáveis foi realizada por meio da análise univariada, com razão de prevalência como medida de associação.

Resultados: A prevalência de sobrepeso encontrada foi de $20 \%$ e a de obesidade de $7 \%$. A maioria dos alunos (72\%) não praticava atividade física fora da escola e pouco mais da metade deles (53\%) gastava mais de quatro horas por dia em atividades sedentárias. $\mathrm{O}$ número de irmãos foi a única variável associada com o excesso de peso $(\mathrm{RP}=1,64$; IC95\% $=1,10-2,45)$ para aqueles que tinham apenas um irmão e $(\mathrm{RP}=1,70$; IC95\%=1,02-2,84) para os que não tinham irmão, quando comparados àqueles que tinham dois ou mais irmãos.

Conclusões: Observou-se elevada prevalência de sobrepeso e obesidade nessa amostra, reforçando a necessidade de mais estudos em crianças residentes em municípios de

Instituição: Programa de Pós-graduação em Ciências da Saúde da Universidade Estadual de Maringá (UEM), Maringá, PR, Brasil

${ }^{1}$ Especialista em Nutrição Clínica pela Universidade Federal do Paraná (UFPR), Maringá, PR, Brasil

${ }^{2}$ Doutora em Filosofia da Enfermagem pela Universidade Federal de Santa Catarina (UFSC); professora da UEM, Maringá, PR, Brasil

${ }^{3}$ Mestre em Ciências da Saúde pela UEM, Maringá, PR, Brasil

${ }^{4}$ Mestrando do Programa de Pós-graduação em Ciências da Saúde da UEM, Maringá, PR, Brasil

${ }^{5}$ Graduanda em Sociologia da UEM, Maringá, PR, Brasil

${ }^{6}$ Doutora em Estatística pela UFSC; professora da UEM, Maringá, PR, Brasil pequeno porte, assim como de pesquisas e ações que visem prevenir e/ou tratar a obesidade.

Palavras-chave: saúde da criança; índice de massa corporal; sobrepeso; obesidade.

\section{ABSTRACT}

Objective: To identify the prevalence of overweight and obesity and associated factors among six to ten-year-old students from public county schools in the urban area of Marialva in Paraná, Brazil.

Methods: Cross-sectional study that used a stratified random sample of 356 students. The following variables were evaluated: body mass index, gender, age, number of siblings, physical activity and sedentary activities. Descriptive statistics was used to verify the distribution of the sample in the strata. The chi-square test was used in order to verify the differences between the distribution of the sample for each category of variables. The association between variables was tested by univariate analysis.

Results: Prevalence of overweight was $20 \%$ and obesity, $7 \%$. Most students (72\%) did not practice physical activity outside school and about half of them (53\%) spent more than four hours a day in sedentary activities. The number of siblings was the only variable associated with excessive weight for those who had only one sibling $(\mathrm{PR}=1.64$; $95 \% \mathrm{CI}=1.10-2.45)$ and for those who had no siblings $(\mathrm{PR}=1.70 ; 95 \% \mathrm{CI}=1.02-2.84)$, compared to those who had two or more siblings.

Endereço para correspondência:

Sonia Silva Marcon

Rua Jailton Saraiva, 526 - Jardim América

CEP 87045-3000 - Maringá/PR

E-mail: soniasilva.marcon@gmail.com

Conflito de interesse: nada a declarar

Recebido em: 2/2/09

Aprovado em: 15/7/09 
Conclusions: There was a high prevalence of overweight and obesity in this sample. Further studies are needed in order to investigate the prevalence of this disease in children living in small Brazilian municipalities, as well as for research and actions to prevent and/or treat obesity.

Key-words: child health; body mass index; overweight; obesity.

\section{Introdução}

Nos últimos anos, houve em aumento significativo na incidência da obesidade infantil em vários países. Esse fato levou a Organização Mundial de Saúde (OMS) a considerar a obesidade uma questão de saúde pública, visto que, além de alterar a imagem pessoal, expõe o indivíduo a uma série de problemas cardiovasculares, respiratórios, musculoesqueléticos e metabólicos ${ }^{(1,2)}$.

A obesidade é o acúmulo generalizado de gordura corporal que decorre de influências genéticas, psicológicas e ambientais, associando-se ao estilo de vida e aos hábitos alimentares, relacionada diretamente à prática de atividade física ${ }^{(3)}$. Crianças obesas são mais suscetíveis a se tornarem adultos obesos e a desenvolverem diabetes melito tipo 2, hipertensão arterial, dislipidemias, aterosclerose, osteoartrite e alguns tipos de neoplasias relacionadas à obesidade $e^{(1,2)}$.

Apesar de fatores genéticos predisporem os indivíduos ao sobrepeso e à obesidade, vários estudos ${ }^{(4-6)}$ têm demonstrado que os hábitos alimentares e a inatividade física parecem interferir de maneira mais significativa sobre a composição corporal, levando o Comitê de Especialistas ${ }^{(7)}$ a recomendar, para prevenir a obesidade, hábitos alimentares saudáveis e prática de atividade física, além da retirada da televisão do quarto das crianças e adolescentes.

Conforme os relatos da OMS, a prevalência de obesidade infantil cresceu em torno de 10 a $40 \%$ na maioria dos países europeus nos últimos dez anos ${ }^{(8)}$. Nos Estados Unidos, a prevalência de sobrepeso dobrou em crianças de seis a 11 anos entre o National Health and Nutrition Examination Survey conduzido em 1999 e 2002, estimando-se que $31 \%$ das crianças dessa faixa etária estavam acima do peso $^{(9)}$. No Brasil, diversos estudos têm sido realizados a esse respeito. No Recife, por exemplo, observou-se uma prevalência de $2,5 \%$ de sobrepeso em crianças e adolescentes de baixa condição socioeconômica, comparado a $10,6 \%$ nos de boa condição socioeconômica ${ }^{(1)}$. No município do Rio de Janeiro, verificou-se $5 \%$ de obesidade em estudantes com idade inferior a dez anos ${ }^{(10)} \mathrm{e}$, em um estudo multicêntrico utilizando os dados da pesquisa sobre padrões de vida realizada pelo Instituto Brasileiro de Geografia e Estatística (IBGE) ${ }^{(11)}$ nas regiões Nordeste e Sudeste, a prevalência de obesidade foi de 8,2 e 11,9\%, respectivamente ${ }^{(2)}$.

No entanto, o relatório da Pesquisa de Orçamentos Familiares 2002-2003 (POF) do IBGE ${ }^{(12)}$ destaca a importância de avaliações regionais, tendo em vista as diferenças esperadas em um país com grande diversidade como o Brasil. Com base nisso e considerando a importância do diagnóstico e tratamento do excesso de peso ainda na infância ${ }^{(13)}$ e, também, o fato de a maioria dos estudos sobre prevalência de sobrepeso e obesidade terem sido realizados em grandes centros ou pelo menos em municípios com mais de $100 \mathrm{mil}$ habitantes, entende-se ser importante estudar a prevalência dessa doença em cidades de pequeno porte e em faixas etárias precoces. Diante disso, o objetivo deste trabalho foi identificar a prevalência de sobrepeso e obesidade nos alunos de seis a dez anos das escolas públicas municipais da área urbana de Marialva, no Paraná, Brasil.

\section{Métodos}

Realizou-se um estudo transversal com alunos de ambos os sexos, entre seis e dez anos de idade, matriculados em escolas públicas municipais da região urbana de Marialva, Paraná, selecionados aleatoriamente.

O município de Marialva possui uma área de $475,55 \mathrm{~km}^{2}$ e é constituído por uma população de 28.702 habitantes, dos quais 22.113 são moradores urbanos ${ }^{(12)}$. O referido município apresenta agricultura bem desenvolvida e se destaca pela produção de uva, sendo conhecido como a Capital da Uva Fina e possuindo, de acordo com a ONU, um IDH médio (0,784). A rede municipal de ensino público possui oito escolas, três situadas na área rural e cinco na área urbana.

Para a seleção das escolas a serem incluídas no estudo, primeiramente procurou-se caracterizar a região onde cada uma das cinco escolas urbanas estava localizada, a partir do levantamento do valor médio do Imposto Predial e Territorial Urbano (IPTU) da região. Esse levantamento mostrou a existência de três padrões de valor; portanto, de acordo com o valor do IPTU, a cidade de Marialva poderia ser dividida em três regiões, devendo ser incluída no estudo apenas uma escola de cada região, visto que atendiam a uma população com características socioeconômicas a priori semelhantes. Em duas regiões havia mais de uma escola 
municipal e, nesses casos, foi feito um sorteio simples de qual seria incluída no estudo.

A amostra foi calculada levando em consideração a prevalência do evento (excesso de peso), o tamanho da população, o nível de confiança e o erro permitido para a diferença máxima entre o resultado da amostra e os valores reais das frequências estudadas ${ }^{(14)}$. A Secretaria de Educação e Cultura de Marialva forneceu a relação do número total de alunos matriculados entre a primeira e a quarta séries na rede pública de ensino, no segundo semestre do ano letivo de 2007 (1.736 alunos). A prevalência de sobrepeso/obesidade foi estimada em $50 \%$, a fim de se garantir a variabilidade máxima da amostra ${ }^{(15)}$ para um nível de confiança de $95 \%$ e erro de $5 \%$. Dessa forma, a amostra deveria contar com 315 escolares, acrescendo-se $20 \%$ para cobrir prováveis perdas de sujeitos e/ou informações, ficando a amostra final planejada para 378 escolares.

Para definir o número de alunos de cada escola a serem incluídos no estudo, fez-se estratificação proporcional ao número de alunos matriculados em cada uma das três escolas em estudo. Em seguida, foi solicitado às escolas selecionadas uma relação, com nome completo e idade, de todos os alunos matriculados da primeira à quarta série por série e turno. Com base nessas informações, foram sorteados aleatoriamente os alunos de cada série a serem incluídos no estudo ${ }^{(16)}$.

A Direção das escolas se encarregou de encaminhar aos pais dos alunos sorteados para participarem do estudo uma carta explicativa sobre os objetivos da pesquisa e o tipo de participação desejada, acompanhada de duas vias do termo de consentimento livre e esclarecido (TCLE) a ser assinado pelos responsáveis.

A coleta de dados foi realizada por duas nutricionistas e o registro foi feito por uma acadêmica do curso de Ciências Sociais na própria escola, durante o horário de aula. Os alunos eram liberados da sala de aula em grupos de cinco. Foram coletadas informações sobre as variáveis: idade (data de nascimento), sexo, peso corporal, altura, prática de atividade física fora da escola e tempo dispensado com atividades sedentárias como assistir televisão, jogar video game e usar o computador.

O peso corporal dos alunos foi aferido por meio de uma balança digital da marca Plenna com capacidade de $150 \mathrm{~kg}$ e precisão de $100 \mathrm{~g}$, com o avaliado na posição ortostática no centro da balança, descalço e vestindo roupas leves. A aferição da altura foi realizada com fita métrica inelástica e escala de $0,5 \mathrm{~cm}$, afixada à parede, estando o escolar em posição ereta, pés descalços e unidos, mãos espalmadas sobre as coxas e cabeça ajustada no plano de Frankfurt ${ }^{(17,18)}$.

Com base nessas informações, o índice de massa corpórea (IMC) foi calculado por meio da divisão do peso em $\mathrm{kg} /$ (estatura em m) ${ }^{2}$. Para a classificação do estado nutricional, foram utilizados os pontos de corte de IMC propostos por Cole et al ${ }^{(18)}$, conforme sexo e idade e de acordo com a recomendação da International Obesity Task Force e de pesquisadores nacionais ${ }^{(2,18,19)}$.

As informações sobre o tempo despendido com atividade física fora da escola e com atividades sedentárias foram obtidas com ajuda das coletoras de dados, que traduziam as perguntas para uma linguagem mais simples como: $\mathrm{O}$ que você costuma fazer quando não está na escola? Quando criança respondia, a entrevistadora fazia referência se era isso que ela tinha feito no dia anterior, tentando identificar o máximo de atividades que a criança havia realizado no período em que não estava na escola.

Para a análise dos dados, utilizou-se a estatística descritiva: média, frequência e percentual. A estatística inferencial envolveu o teste $t$ de Student para amostras independentes e o teste do qui-quadrado. A associação entre as variáveis foi testada por meio da análise univariada, que utilizou a razão de prevalência como medida de associação. Todos os dados foram processados e tratados com auxílio do programa Statistica, versão 7.1.

O desenvolvimento do estudo obedeceu aos preceitos da Resolução 196/96 do Conselho Nacional de Saúde, que estabelece normas para pesquisa com seres humanos, e seu projeto foi aprovado pelo Comitê de Ética em Pesquisa com Seres Humanos da Universidade Estadual de Maringá. Todos os alunos envolvidos na pesquisa e seus respectivos responsáveis foram informados quanto aos objetivos do estudo e esclarecidos e quanto aos métodos utilizados na pesquisa, ficando garantido aos participantes o direito de desistir do estudo a qualquer momento. Participaram da amostra apenas os escolares selecionados que retornaram com o TCLE devidamente assinado por seu responsável.

\section{Resultados}

Dos 378 estudantes selecionados, $22(5,8 \%)$ foram excluídos: 16 por não apresentarem o TCLE assinado pelos pais ou responsáveis até o dia da coleta de dados e seis por não estarem presentes no dia agendado para a coleta de dados. Dessa forma, a amostra foi constituída por 356 escolares de 
ambos os sexos, com idade variando entre seis e dez anos. O peso médio observado foi de $34,8 \pm 10,0 \mathrm{~kg}$ para os meninos e $33,9 \pm 8,8 \mathrm{~kg}$ para as meninas. A altura média encontrada foi de $134,5 \pm 9,0$ e $134,8 \pm 8,5 \mathrm{~cm}$ para meninos e meninas, respectivamente. O IMC das meninas $(18,4 \pm 3,2)$ foi significativamente menor do que o dos meninos $(19,0 \pm 3,7)$ $(p=0,039)$.

Na Tabela 1 é apresentada a caracterização dos escolares investigados. Verifica-se que a distribuição da amostra foi significativamente diferente para as variáveis número de irmãos, estado nutricional, atividade física e atividades sedentárias $(p<0,001)$. Não houve diferenças para as variáveis sexo e faixa etária.

A prevalência de sobrepeso e obesidade foi de 20,2 e 7,0\%, respectivamente. Em relação à prática de atividade física fora da escola, $71,9 \%$ dos indivíduos responderam não praticar qualquer atividade física e $53,4 \%$ referiram gastar mais de quatro horas diárias em atividades sedentárias, como assistir televisão, ficar no computador ou jogar video game.

Ao analisar as prevalências de sobrepeso e obesidade de acordo com o sexo e a idade, verificou-se que, na faixa etária de sete a dez anos, a prevalência de sobrepeso é maior nas meninas. A prevalência de obesidade é mais elevada no sexo masculino, em todas as faixas etárias. Houve significância estatística na prevalência de obesidade em relação ao sexo apenas para os escolares de seis anos de idade $(p<0,01)$. Com relação à prática de atividade física fora da escola, observou-se que os alunos de oito a dez anos de idade eram os que despediam mais tempo nessas atividades. A média de tempo gasto com a prática de atividade física foi de $1,1 \pm 0,8 \mathrm{horas} / \mathrm{dia}$.

Quanto às atividades sedentárias, verificou-se que essas crianças passavam em média $3,8 \pm 1,4 \mathrm{~h} /$ dia assistindo televisão e/ou jogando video game e/ou usando o computador. Além disso, 53,4\% dos escolares passavam mais de $4 \mathrm{~h} /$ dia em atividade sedentárias, sendo que, entre esses, a prevalência de sobrepeso e obesidade foi respectivamente de 25,8 e 7,9\%, valores mais altos do que os observados na amostra total, porém sem diferença estatística. O percentual de estudantes com o maior número de horas em atividades sedentárias era relativamente maior entre os meninos $(51,6 \%)$ e, a partir dos sete anos, tal diferença praticamente não existiu.

$\mathrm{Na}$ Tabela 2 é apresentada a associação das variáveis independentes com o excesso de peso. Não foi encontrada associação estatisticamente significativa para nenhuma das variáveis, com exceção à variável "número de irmãos". As crianças que relataram ter apenas um irmão mostraram 6,4 vezes mais chance de excesso de peso, comparadas com seus pares que tinham dois ou mais irmãos. Já as crianças que não tinham irmãos apresentaram 7,0 vezes mais chance de excesso de peso do que as que tinham dois ou mais irmãos.

\section{Discussão}

A prevalência de sobrepeso e obesidade infantil tem sido muito estudada ultimamente; no entanto, a existência de diferentes critérios diagnósticos e pontos de cortes possibilita que uma mesma amostra de referência apresente resultados diversos ${ }^{(17)}$. Além disso, tal prevalência varia muito de um local para outro, pois é condicionada por diversos fatores, tais como: biológicos, psicológicos e socioeconômicos ${ }^{(3)}$.

No presente estudo, foi observada alta prevalência de sobrepeso $(20,2 \%)$ e obesidade $(7,0 \%)$. A prevalência de excesso de peso encontrada em nosso estudo $(27,2 \%)$ é superior à verificada nos Estados Unidos $(25,6 \%)$ e maior que o dobro da verificada no Brasil $(13,6 \%)$ em uma pesquisa de abrangência mundial que mostrou o aumento na prevalência de sobrepeso em indivíduos de seis a 18 anos de idade na maioria dos países investigados ${ }^{(20)}$. Entre os anos de 1999 e 2002, a

Tabela 1 - Caracterização das crianças investigadas em escolas públicas municipais de Marialva (PR)

\begin{tabular}{lrrc}
\hline & $\mathbf{n}$ & $\%$ & Valor de $\boldsymbol{p}$ \\
\hline Sexo & & & 0,711 \\
$\quad$ Masculino & 174 & 48,9 & \\
$\quad$ Feminino & 182 & 51,1 & \\
$\begin{array}{l}\text { Faixa etária } \\
\quad \text { 8 anos }\end{array}$ & 187 & 52,5 & \\
$\quad$ 9 anos & 169 & 47,5 & \\
$\quad$ Número de irmãos & & & $<0,001$ \\
$\quad$ ou mais & 143 & 40,2 & \\
$\quad 1$ irmão & 162 & 45,5 & \\
$\quad$ Nenhum & 51 & 14,3 & \\
Estado nutricional & & & $<0,001$ \\
$\quad$ Eutrofia & 259 & 72,8 & \\
$\quad$ Sobrepeso & 72 & 20,2 & \\
$\quad$ Obesidade & 25 & 7 & \\
Atividade física & & & $<0,001$ \\
$\quad$ Sim & 100 & 28,1 & \\
$\quad$ Não & 256 & 71,9 & \\
Atividades sedentárias & & & $<0,001$ \\
$\quad$ Menos de 1 h/dia & 29 & 8,2 & \\
$\quad$ de 1 a 4 h/dia & 182 & 51,1 & \\
$\quad$ Mais de 4 h/dia & 145 & 40,7 & \\
$\quad$ & & & \\
\hline
\end{tabular}


Tabela 2 - Associação das variáveis independentes com o excesso de peso em crianças investigadas em escolas públicas municipais de Marialva (PR)

\begin{tabular}{|c|c|c|c|c|c|}
\hline \multirow[t]{2}{*}{ Variável } & \multirow{2}{*}{$\mathrm{n}$ total } & Eutróficos & $\begin{array}{c}\text { Excesso de } \\
\text { peso }\end{array}$ & \multirow[t]{2}{*}{$\mathbf{R P}$} & \multirow[t]{2}{*}{ IC95\% } \\
\hline & & $\%$ & $\%$ & & \\
\hline \multicolumn{6}{|l|}{ Sexo } \\
\hline Masculino & 174 & 72,4 & 27,6 & 1 & - \\
\hline Feminino & 182 & 73,1 & 26,9 & 0,98 & $0,70-1,37$ \\
\hline \multicolumn{6}{|l|}{ Faixa etária } \\
\hline$\leq 8$ anos & 187 & 70,6 & 29,4 & 1 & - \\
\hline$\geq 9$ anos & 169 & 75,2 & 24,8 & 0,84 & $0,60-1,19$ \\
\hline \multicolumn{6}{|l|}{ Número de irmãos } \\
\hline 2 ou mais & 143 & 32,3 & 7,9 & 1 & - \\
\hline 1 irmão & 162 & 30,9 & 14,6 & 1,64 & $1,10-2,45$ \\
\hline Nenhum & 51 & 9,6 & 4,8 & 1,7 & $1,02-2,84$ \\
\hline \multicolumn{6}{|l|}{ Atividade física } \\
\hline $\operatorname{Sim}$ & 100 & 20,5 & 7,6 & 1 & - \\
\hline Não & 256 & 52,2 & 19,7 & 1,01 & $0,69-1,48$ \\
\hline \multicolumn{6}{|c|}{ Atividades sedentárias } \\
\hline Menos de 1h/dia & 29 & 6,2 & 2 & 1 & - \\
\hline de 1 a $4 \mathrm{~h} / \mathrm{dia}$ & 182 & 38,5 & 12,6 & 1,02 & $0,51-2,05$ \\
\hline Mais de 4h/dia & 145 & 28,1 & 12,6 & 1,29 & $0,65-2,56$ \\
\hline
\end{tabular}

$\mathrm{RP}=$ razão de prevalência; IC=intervalo de confiança.

prevalência de sobrepeso dobrou para a faixa etária de seis a 11 anos nos Estados Unidos, estimando-se que atualmente esteja em torno de $31 \%{ }^{(9)}$. A maior quantidade de gordura corporal na infância e adolescência é verificada em estudos de tendência secular ${ }^{(20)}$.

Valores semelhantes foram encontrados em outros estudos com escolares da mesma faixa etária, dois deles realizados em municípios próximos ao de Marialva: um em Apucarana (PR), no qual foi encontrado $20,2 \%$ de sobrepeso e $4,3 \%$ de obesidade $^{(21)}$, e outro que abordou apenas alunos de elevado nível socioeconômico matriculados em uma instituição privada de Londrina (PR), no qual foi relatado $18,5 \%$ de sobrepeso ${ }^{(22)}$.

As prevalências de sobrepeso e obesidade foram relativamente maiores do que as encontradas em estudo realizado com alunos da primeira e segunda séries do Ensino Fundamental na Vila Mariana, em São Paulo (SP) (10,9 e 8,2\%, respectivamente) ${ }^{(23)}$, em alunos de seis a dez anos de uma escola privada de Brasília $\left(14,6\right.$ e 5,5\%) ${ }^{(17)}$ e entre alunos de sete a nove anos de uma escola pública de Florianópolis (17,9 e 6,7\%) ${ }^{(24)}$. Já em estudo realizado com estudantes de escolas municipais na cidade do Rio de Janeiro, com idade inferior a dez anos, observou-se prevalência de $5 \%$ de obesidade ${ }^{(10)}$.

Chama atenção o fato de que, independentemente da região em que os diferentes estudos foram realizados, identifica-se uma alta prevalência de sobrepeso e obesidade em crianças e adolescentes. É importante ressaltar que apenas no estudo realizado na Vila Mariana ${ }^{(23)}$ a prevalência de obesidade foi maior do que a encontrada neste estudo $(8,2 \%)$, o que é bastante preocupante, pois esperava-se que a prevalência de excesso de peso fosse mais baixa em municípios de pequeno porte pela falta de "liberdade" que as crianças de grandes centros têm para realizarem atividades fora de casa e ao ar livre.

À análise das prevalências de sobrepeso e obesidade de acordo com o sexo e a faixa etária, observa-se que o sobrepeso é maior entre as meninas de sete a dez anos; esses achados confirmam a tendência observada nos estudos realizados em Apucarana (PR) ${ }^{(21)}$ e no Rio de Janeiro, que identificaram ser a prevalência de sobrepeso em meninas mais elevada em todas as faixas etárias ${ }^{(10)}$.

Quanto à obesidade, foi verificada maior prevalência no sexo masculino em todas as faixas etárias, porém houve significância estatística apenas nos escolares de seis anos $(p<0,01)$. Tais achados se aproximam dos encontrados em estudo realizado em Londrina $(\mathrm{PR})^{(22)}$, no qual os meninos apresentaram maior prevalência de obesidade em todas as faixas etárias, embora tenha sido observada significância estatística nas faixas etárias de nove a dez anos de idade $(p<0,05)$. Cabe ressaltar que o sexo não se mostrou relevante para sobrepeso 
e nem para obesidade em estudo realizado com estudantes de seis a 14 anos na região Sul de São Paulo(25).

A participação da criança em atividades desportivas é parte do processo de socialização, pois, além dos benefícios para a saúde, oferece oportunidade de lazer e desenvolvimento de aptidões que melhoram a autoestima e a confiança ${ }^{(26)}$. No entanto, a prática de atividade física fora da escola foi relatada por apenas $28,1 \%$ dos alunos, resultado muito inferior ao percentual encontrado em escolares franceses de sete a nove anos de idade (66,5\%). Entretanto, está próxima dos valores relatados por escolares de Florianópolis $(35,7 \%)$ na mesma faixa etária ${ }^{(27)}$ e, também, por estudantes de Goiânia ${ }^{(27)}$, apesar de a faixa etária estudada ser mais ampla (sete a 14 anos).

No presente estudo, o tempo médio despendido com atividade física fora da escola foi de $1,1 \pm 0,8$ horas. Pesquisadores de Chicago, Estados Unidos, encontraram forte correlação negativa entre IMC e tempo de atividade física $(p<0,0001)$, sugerindo que a obesidade seria o maior contribuinte para o decréscimo de atividade física em uma amostra de 525 crianças e adolescentes de quatro a 18 anos de idade que moram em área urbana ${ }^{(28)}$.

O tempo médio gasto em atividades sedentárias - como assistir televisão e/ou jogar video game e/ou usar o computador - foi de 3,8 $\pm 1,4 \mathrm{~h} /$ dia. Porém, não houve associação entre as atividades sedentárias e o excesso de peso, o que é discordante dos achados de outras pesquisas ${ }^{(29,30)}$. No estudo realizado na zona Sul de São Paulo, por exemplo, os indivíduos com sobrepeso e obesidade foram os que menos praticavam esporte durante a semana ${ }^{(25)}$. É interessante observar que, embora o estudo não tenha avaliado o consumo alimentar das crianças, outros autores demonstram que o fato de permanecer tempo em demasia assistindo à televisão, jogando video game e/ou em frente ao computador é agravado pelo consumo de alimentos pouco nutritivos e de alta densidade energética, muitas vezes induzidos pelas propagandas exibidas nos intervalos da programação infantil em geral ${ }^{(3,31)}$.

As crianças que têm até um irmão apresentaram maiores chances de excesso de peso, o que merece maior atenção em pesquisas futuras. No presente estudo, tal achado não poderia ser explicado pelo fato dessas crianças não adotarem hábitos alimentares saudáveis, visto que os hábitos alimentares não foram investigados, nem pelo fato de serem adeptas a atividades mais sedentárias, já que não foi observada associação entre atividade física e excesso de peso.

Esta pesquisa contribui para o entendimento da prevalência de sobrepeso e obesidade em cidade de pequeno porte e com crianças em idades precoces, características pouco estudadas; entretanto, constituiu uma limitação do estudo a não-investigação dos hábitos alimentares e o fato de informações importantes - como o tempo gasto com a prática de atividade física e em atividades sedentárias - terem sido coletadas diretamente com as crianças, considerando que estas ainda se encontram no início da vida escolar, podendo provocar algum tipo de viés nos resultados obtidos.

Portanto, sugere-se que novas pesquisas sejam realizadas com obtenção apropriada de informações referentes à prática de atividade física e tempo gasto em atividades sedentárias, além dos hábitos alimentares, de forma que se possam fazer inferências mais seguras dos fatores associados ao excesso de peso em populações com essas características.

Os resultados encontrados revelam elevada prevalência de sobrepeso e obesidade nos escolares investigados, e isso aponta que, além da necessidade de mais estudos de prevalência dessa doença em crianças residentes em municípios de pequeno porte, é imperativa a união de esforços por parte de diferentes segmentos, mas principalmente da escola e do setor de saúde, para implementar medidas para prevenir e/ ou tratar a obesidade.

\section{Referências bibliográficas}

1. Silva GA, Balaban G, Motta ME. Prevalence of overweight and obesity in children and adolescents of different socioeconomic conditions. Rev Bras Saude Matern Infant 2005;5:53-9.

2. Abrantes MM, Lamounier JA, Colosimo EA. Overweight and obesity prevalence among children and adolescents from Northeast and Southeast regions of Brazil. J Pediatr (Rio J) 2002;78:335-40.

3. Oliveira AM, Cerqueira EM, Souza JS, Oliveira, AC. Sobrepeso e obesidade infantil: influência de fatores biológicos e ambientais em Feira de Santana, BA. Arq Bras Endocrinol Metab 2003;47:144-50.
4. Delmas C, Platat C, Schweitzer B, Wagner A, Oujaa M, Simon C. Association between television in bedroom and adiposity throughout adolescence. Obesity (Silver Spring) 2007;15:2495-503.

5. Waller K, Kaprio J, Kujala UM. Associations between long-term physical activity, waist circumference and weight gain: a 30-year longitudinal twin study. Int $\mathrm{J}$ Obes (Lond) 2008;32:353-61.

6. Branca F, Nikogosian H, Lobstein T. The challenge of obesity in the WHO European Region and the strategies for response. Geneva: WHO Europe; 2007. 
7. Barlow SE; Expert Committee. Expert committee recommendations regarding the prevention, assessment, and treatment of child and adolescent overweight and obesity: summary report. Pediatrics 2007;120:S164-92.

8. Ebbeling CB, Pawlak DB, Ludwig DS. Childhood obesity: public-health crisis, common sense cure. Lancet 2002;360:473-82.

9. Hedley AA, Ogden CL, Johnson CL, Carroll MD, Curtin LR, Flegal KM. Prevalence of overweight and obesity among US children, adolescents, and adults, 1999-2002. JAMA 2004;291:2847-50.

10. Anjos LA, Castro IR, Engstrom EM, Azevedo AM. Growth and nutritional status in a probabilistic sample of schoolchildren from Rio de Janeiro, 1999. Cad Saude Publica 2003;19:S171-9.

11. Brasil. Ministério do Planejamento, Orçamento e Gestão. Pesquisa sobre padrões de vida 1996-1997. 2a ed. Rio de Janeiro: IBGE; 1999.

12. Brasil. Ministério do Planejamento, Orçamento e Gestão. Pesquisa de Orçamentos Familiares 2002-2003. Antropometria e análise do estado nutricional de crianças e adolescentes no Brasil. Rio de Janeiro: IBGE; 2006.

13. Taylor RW, Jones IE, Williams SM, Goulding A. Evaluation of waist circumference, waist-to-hip ratio, and the conicity index as screening tools for high trunk fat mass, as measured by dual-energy $\mathrm{X}$-ray absorptiometry, in children aged 3-19 y. Am J Clin Nutr 2000;72:490-5.

14. Silva NN. Amostragem probabilística: um curso introdutório. 2a ed. São Paulo: Edusp; 2004.

15. Tanaka OY, Espírito Santo ACG. Avaliação da qualidade da atenção básica utilizando a doença respiratória da infância como traçador, em um distrito sanitário do município de São Paulo. Rev Bras Saude Matern Infant 2008;8:325-32.

16. Pocock SJ. Clinical trials: a pratical approach. Chichester: Wiley; 1989.

17. Giugliano R, Melo AL. Diagnóstico de sobrepeso e obesidade em escolares: utilização do índice de massa corporal segundo padrão internacional. J Pediatr (Rio J) 2004;80:129-34.

18. Cole TJ, Bellizzi MC, Flegal KM, Dietz WH. Establishing a standard definition for child overweight and obesity worldwide: international survey. BMJ 2000;320:1240-3.

19. de Assis MA, Rolland-Cachera MF, Grosseman S, de Vasconcelos FA, Luna ME, Calvo MC et al. Obesity, overweight and thinness in schoolchildren of the city of Florianópolis, Southern Brazil. Eur J Clin Nutr 2005;59: 1015-21.

20. Wang Y, Monteiro C, Popkin BM. Trends of obesity and underweight in older children and adolescents in the United States, Brazil, China, and Russia. Am J Clin Nutr 2002;75:971-7.

21. Guedes DP, Paula IG, Guedes JE, Stanganelli LC. Prevalência de sobrepeso e obesidade em crianças e adolescentes: estimativas relacionadas ao sexo, à idade e à classe socioeconômica. Rev Bras Educ Fis Esp 2006;20:151-63.

22. Ronque ER, Cyrino ES, Dórea VR, Serrasuelo HJ, Galdi EHG, Arruda $M$. Prevalência de sobrepeso e obesidade em escolares de alto nivel socioeconômico em Londrina, Paraná, Brasil. Rev Nutr Campinas 2005;18:709-17.

23. Sotelo YO, Colugnati FA, Taddei JA. Prevalência de sobrepeso e obesidade entre escolares da rede pública segundo três critérios de diagnóstico antropométrico. Cad Saude Publica 2004;20:233-40.

24. Soar C, Vasconcelos FA, Assis MA, Grosseman S, Luna ME. Prevalence of overweight and obesity in school children in public school of Florianópolis, Santa Catarina. Rev Bras Saude Matern Infant 2004;4:391-7.

25. Fagundes AL, Ribeiro DC, Naspitz L, Garbelini LE, Vieira JK, Silva AP et al. Prevalence of overweight and obesity in school children of Parelheiros region in São Paulo city, Brazil. Rev Paul Pediatr 2008;26:212-7.

26. Juswiak CR, Paschoal VC, Lopez FA. Nutrição e atividade física. J Pediatr 2000;76:349-58

27. Assis MA, Rolland-Cachera MF, Vasconcelos FA, Bellisle F, Calvo MC, Luna ME et al. Overweight and thinness in 7-9 year old children from Florianópolis, Southern Brazil: a comparison with a French study using a similar protocol. Rev Nutr Campinas 2006;19:299-308.

28. Chatrath R, Shenoy R, Serratto M, Thoele DG. Physical fitness of urban American children. Pediatr Cardiol 2002;23:608-12.

29. Arluk SL, Branch JD, Swain DP, Dowling EA. Childhood obesity's relationship to time spent in sedentary behavior. Mil Med 2003;168:583-6.

30. Brown D. Playing to win: video games and the fight against obesity. J Am Diet Assoc 2006;106:188-9.

31. Francis $L A$, Lee $Y$, Birch LL. Parental weight status and girls' television viewing, snacking, and body mass indexes. Obes Res 2003;11:143-51. 